\title{
Gli autori
}

Maria Francesca Alfonsi è giornalista Rai.

Carlo Bisci è professore associato in Geografia fisica e Geomorfologia, Università di Camerino.

Elena Bitocchi è assegnista di ricerca in Agronomia e Genetica Agraria, Università Politecnica delle Marche.

Maria Grazia Camilletti è docente nelle scuole superiori; è stata presidente dell'Istituto Storia Movimento Liberazione Marche.

Emilio D'Alessio è architetto, pianificatore, esperto in sviluppo sostenibile.

Roberto Danovaro è professore ordinario di Ecologia, Università Politecnica delle Marche.

Anna Elisa De Gregorio è una scrittrice.

Roberto Deidda è professore associato in Costruzioni Idrauliche, Marittime e Idrologia, Università di Cagliari.

Roberta Farina è ricercatrice presso il Consiglio per la Ricerca in Agricoltura (CRA), Foggia.

Patrizia Gabrielli è professore ordinario di Storia contemporanea e Storia delle donne e delle relazioni di genere, Università di Siena.

Benedetta Giovanola è ricercatrice in Filosofia Morale, Università di Macerata.

Katiuscia Grassi lavora presso il Servizio Territorio Ambiente Energia, Regione Marche.

Valerio Lucarini è lecturer in Meteorology and Mathematics, Università di Reading (UK).

Roberto Papa è direttore del Centro di ricerca per la cerealicoltura del CRA, Foggia, e professore associato di Genetica Agraria, Università Politecnica delle Marche.

Luigino Quarchioni è presidente di Legambiente Marche.

Alessandro Sterlacchini è professore ordinario in Economia applicata, Università Politecnica delle Marche. 


\section{PRISMA. NORME EDITORIALI}

\section{Politica editoriale}

La Rivista costituisce uno spazio di analisi critica e di riflessione sulle trasformazioni economiche e sociali che coinvolgono direttamente o indirettamente la realtà marchigiana, e in particolare il mondo del lavoro, anche tramite l'indagine comparata con altre realtà regionali.

I temi oggetto dei singoli fascicoli sono affrontati con un taglio di tipo monografico e interdisciplinare. La combinazione di contributi di natura teorica e di riferimento generale, con articoli empirici e direttamente riguardanti il territorio marchigiano, allarga l'orizzonte di interesse dall'ambito locale a quello nazionale ed europeo.

In ogni numero, inoltre, viene pubblicato un saggio "Fuori dal tema" non vincolato ai temi monografici.

Infine, la presenza di forum di discussione, aperti ai contributi delle forze sociali e politiche, permette alla rivista di ospitare un confronto tra le diverse realtà sociali, favorire una crescita consapevole della cittadinanza ed essere strumento metodologico in mano al decision maker, promuovendo un dibattito sulle ipotesi emergenti dai contributi critici e orientando prassi più consapevoli.

\section{Consegna dei manoscritti}

I manoscritti dovranno essere inviati alla redazione della Rivista, all'indirizzo di posta elettronica prisma@iresmarche.net, e dovranno essere accompagnati da un breve profilo dell'autore (contenente alcune notizie biografiche essenziali, corredate da affiliazione, indirizzo, numero di telefono e indirizzo e-mail) e da un abstract in lingua inglese (massimo 170 parole).

Nell'oggetto dell'e-mail dovrà essere indicato: "Proposta di saggio fuori dal tema", oppure "Proposta di saggio per il numero monografico n. «X, anno Y»".

I saggi pervenuti verranno valutati da due referees anonimi: pertanto si invita a evitare, nel manoscritto, ogni riferimento o indicazione che possa ricondurre all'autore (es. invece di scrivere "come ho argomentato in Accornero, 1980", utilizzare l'espressione "come è stato argomentato in Accornero, 1980").

Gli autori riceveranno comunicazione dell'esito del referaggio entro 45 giorni dalla ricezione da parte della redazione e, una volta acquisiti i commenti dei referees, avranno 2 settimane di tempo per apportare le eventuali modifiche richieste.

Gli articoli devono essere accompagnati da una lettera di liberatoria in cui l'autore concede alla Direzione della rivista l'esercizio esclusivo di tutti i diritti di sfruttamento economico sul'articolo, senza limiti di spazio ed entro i limiti temporali massimi riconosciuti dalla normativa vigente (attualmente 20 anni) e con tutte le modalità e le tecnologie attualmente esistenti e/o in futuro sviluppate. Si intende pertanto compresa, inter alia, la riproduzione in ogni modo e forma, comunicazione - ivi inclusi, a titolo esemplificativo ma non esaustivo, anche i diritti di sfruttamento patrimoniale a mezzo di canali digitali interattivi (con qualsiasi modalità attualmente nota od in futuro sviluppata) - e distribuzione di cui l'articolo è su- 
scettibile. Parimenti concede alla Direzione in esclusiva il diritto di tradurre, riprodurre, distribuire, comunicare l'articolo in qualsiasi lingua, in qualsiasi modo e forma, i diritti di sfruttamento pubblicitario, il diritto di cedere a terzi i diritti loro spettanti sull'articolo, in tutto o in parte, sia a titolo oneroso che a titolo gratuito, ecc., senza limiti di spazio ed entro i limiti temporali massimi riconosciuti dalla normativa vigente (attualmente, 20 anni) e con tutte le modalità e tecnologie attualmente esistenti e/o in futuro sviluppate.

\section{Norme editoriali}

Prisma è una rivista pensata per un pubblico di non soli "addetti ai lavori”. Pertanto si invitano gli autori a scrivere nel modo più chiaro possibile, evitando uno stile troppo specialistico; ad aprire il saggio con una breve introduzione che illustri la tesi di fondo e a concluderlo con una nota di sintesi che ne riassuma le principali evidenze. È inoltre preferibile limitare il numero delle indicazioni bibliografiche (massimo 10), delle note e dei grafici e/o figure.

Formato e lunghezza del testo. Il testo può avere una lunghezza massima di 45.000 caratteri (spazi inclusi). Tale limite si intende comprensivo di note a piè pagina e riferimenti bibliografici. Qualora l'articolo presenti grafici e figure, il limite delle 45.000 battute si intende ridotto per fare spazio al testo. Articoli che eccedano tale limite non verranno presi in considerazione. Il testo deve essere corredato inoltre da un abstract in inglese di non più di 170 parole.

Il testo deve essere inviato in formato .doc o .rtf. Per la formattazione del testo va utilizzato il carattere Times New Roman, corpo: pt. 10; note a piè pagina: pt. 8. Sul sito della Rivista (http://www.iresmarche.net/) è comunque presente un modello di pagina da utilizzare per la stesura del proprio manoscritto.

Tabelle, Figure, Grafici e Diagrammi. Per tabelle, figure, grafici e diagrammi è richiesta l'indicazione della fonte. Vanno inoltre evitati i colori. Eventuali pattern devono essere molto marcati per dare un buon risultato in sede di stampa. Tabelle, figure, grafici e diagrammi vanno inseriti direttamente nel corpo del testo con un chiaro richiamo (es.: Tabella 1). Il titolo va scritto con il carattere Arial, pt. 8, stile: normale. La fonte va citata con il carattere Arial, pt. 8, stile: corsivo.

Riferimenti bibliografici. I riferimenti bibliografici vanno inseriti nel testo citando solo il cognome dell'autore, l'anno di pubblicazione ed eventualmente le pagine citate. Per esempio: (Accornero, 1980, p. 7).

Nel caso di due autori, vanno citati entrambi i cognomi, per esempio: Frey - Stutzer, 2006. Nel caso di più di due autori, va riportato il primo cognome seguito da et al. Alla fine del testo i riferimenti bibliografici devono essere indicati in ordine alfabetico. Più opere dello stesso autore vanno elencate cominciando dalla più recente. I riferimenti bibliografici alla fine del testo vanno indicati come segue:

Accornero A., (1980) Il lavoro come ideologia, il Mulino, Bologna

Sen A., (1980) "Labour and Technology", in J. Cody, H. Hughes e D. Walls (a cura di), Policies for Industrial Progress in Developing Countries, Oxford University Press, New York, pp. 121-158

Sen A., (1985) "Well-Being, Agency, Freedom. The Dewey Lectures 1984", The

Journal of Philosophy, vol. 82, pp. 169-221 